\title{
Frailty in Older Adults with Mild Dementia: Dementia with Lewy Bodies and Alzheimer's Disease
}

\author{
Miguel Germán Borda ${ }^{a-c}$ Hogne Soennesyn ${ }^{a}$ Claire J. Steves ${ }^{d}$ \\ Audun Osland Vik-Mo a, e Mario Ulises Pérez-Zepedab, f, g Dag Aarslanda, h \\ ${ }^{a}$ Centre for Age-Related Medicine (SESAM), Stavanger University Hospital, Stavanger, \\ Norway; bemillero de Neurociencias y Envejecimiento, Ageing Institute, Medical School \\ Pontificia Universidad Javeriana, Bogotá, Colombia; ${ }^{C}$ Faculty of Health Sciences, University of \\ Stavanger, Stavanger, Norway; ${ }^{\mathrm{d} D e p a r t m e n t}$ of Twin Research and Genetic Epidemiology, \\ King's College London, London, UK; ${ }^{e}$ Department of Clinical Science, University of Bergen, \\ Bergen, Norway; ${ }^{f}$ Geriatric Epidemiology Research Department, Instituto Nacional De \\ Geriatría, Mexico, Mexico; ${ }^{9}$ Geriatric Medicine Research Unit, Department of Medicine, \\ Dalhousie University, Halifax, NS, Canada; ${ }^{\text {h}}$ Department of Old Age Psychiatry, Institute of \\ Psychiatry, Psychology and Neuroscience, King's College London, London, UK
}

\section{Keywords}

Frailty · Dementia, Alzheimer's disease - Dementia with Lewy bodies

\begin{abstract}
Introduction: The aim of the study is to describe the frequency of frailty in people with a new diagnosis of mild dementia due to Alzheimer's disease (AD) and dementia with Lewy bodies (DLB). Methods: This is a secondary analysis of the Dementia Study of Western Norway (Demvest). For this study, we analysed a sample of 186 patients, 116 with AD and 70 with DLB. Subjects were included at a time in which mild dementia was diagnosed according to consensus criteria after comprehensive standardized assessment. Frailty was evaluated retrospectively using a frailty index generated from existing data. The cut-off value used to classify an older adult as frail was 0.25 . Results: The prevalence of frailty was $25.81 \%(n=48)$. In the DLB group, $37.14 \%(n=26)$ were classified as frail, compared to $18.97 \%(n=22)$ of those with AD ( $p<$ 0.001). The adjusted multivariate analysis revealed an OR of 2.45 (1.15-5.23) for being frail in those with DLB when using AD as the reference group. Conclusion: Frailty was higher than expected in both types of dementia. The prevalence of frailty was higher in those with DLB compared to AD. This new finding underscores the need for a multi-systems approach in both dementias, with a particular focus on DLB.




\section{Introduction}

Frailty leads to increased vulnerability toward stressors in older adults and is a known predictor for several adverse outcomes in this age group [1]. The most popular criteria used in order to operationalize frailty are the so-called frailty phenotype and the Frailty Index (FI) [2-4]. Studies suggest that around $9-17 \%$ of community-dwelling older adults over the age of 60 are frail $[5,6]$. However, estimates vary depending on the criteria used for defining said condition [6].

With demographic transition, the number of older adults is increasing, and with it the prevalence of chronic conditions. Dementia is considered one of these [7], known for its terminal and disabling nature, high costs for healthcare systems, and serious consequences for both patients and caregivers [7-9]. This condition has traditionally been considered to be independent from purely physical domains. However, there is enough evidence to infer that both frailty and dementia are main drivers of disability related to aging, as well as for increased mortality [10, 11]. Dementia affects several systems and leads to many manifestations beyond cognition. For instance, it has been associated with geriatric syndromes [12]. Frailty and dementia are multifactorial in origin and share common etiological pathways [12]. Thus, both have been conceptualized as converging conditions resulting from cumulative exposure over time [13].

Previous studies have examined the relationship between frailty and cognitive impairment with a special focus on Alzheimer's disease (AD) [14], but none have studied other highly disabling and frequent dementias such as DLB (Dementia with Lewy Bodies). DLB is the second most common neurodegenerative dementia and is characterized by a tetrad consisting of signs and symptoms beyond cognitive impairment [15]. DLB patients have poor prognosis on a variety of key outcomes, including frequency and duration of hospital admissions, the need for specialized care, under-diagnosis, institutionalization, geriatric syndromes, autonomic impairment, behavioral disturbances, decreased quality of life, and increased caregiver burden [16-18].

The aim of this study is to describe the frequency of frailty in subjects with newly diagnosed mild DLB and mild AD. Given the lack of knowledge on frailty in DLB, it is important to estimate the frequency of said condition in this population, since understanding this association has the potential for better management and better outcomes.

\section{Materials and Methods}

\section{Setting and Participants}

We analyzed data from the Dementia Study of Western Norway (Demvest). This is a longitudinal cohort study with annual assessment of patients referred to dementia clinics in Hordaland and Rogaland counties [19]. Only baseline data were included in this study. The centers were contacted by certified mail prior to the beginning of the study and invited to refer all patients with suspected dementia. All dementia diagnostic units in the region recruited patients to the study. The same National Insurance Scheme with restricted copayments covered all residents of the region. Thus, it was possible to adequately represent dementia in the general population. After the main inclusion period, which took place between 2005 and 2007, we continued to selectively recruit patients with DLB dementia in order to enhance the number of patients in this group.

Exclusion criteria were the absence of dementia, having moderate or severe dementia, delirium, previous bipolar or psychotic disorder, terminal illness, or recently diagnosed major somatic illness, which might significantly impact on cognition, function, or study participation.

After screening 657 patients, 234 were included at baseline. The sample included 116 subjects with AD, 70 with DLB, 12 with Parkinson's disease, 20 had mixed vascular and AD 
and 16 participants had other types of dementia (frontotemporal dementia $n=4$, vascular dementia $n=9$, and alcohol-related dementia $n=3$ ] [19]. For the purposes of our study, we included subjects living with AD and DLB, yielding a total of 186 participants. The youngest subject was 50 years old and the oldest was 92 years of age.

\section{Clinical Assessments}

The diagnosis of dementia was made according to DSM-IV criteria. It was further classified as AD dementia when complying with The National Institute of Neurological and Communicative Disorders and Stroke-AD and Related Disorders Association guidelines [20] and as DLB according to the revised consensus criteria [21]. Two of the study authors independently applied the diagnostic criteria. In cases of disagreement, and in patients fulfilling more than one set of operationalized diagnostic criteria, the final ascertainment was made through consensus. In addition, based on the same evaluation, the consensus criteria for a clinical diagnosis of probable DLB were also applied, and ultimately the proportion fulfilling the 2 sets of criteria for DLB were compared [19]. Mild dementia was defined as a Mini-Mental Status Examination (MMSE) score [22] of at least 20 or a CDR global score $=1$. A pathological diagnosis was available for 56 patients in the Demvest cohort, proving diagnostic accuracy above $80 \%$ for both AD and DLB $[19,23]$.

Patients were evaluated through structured and detailed assessments; in addition, relevant information regarding past medical history was taken from medical records in order to obtain complete and detailed information about the medical background and comorbidities for each subject. Routine blood and CSF analyses, as well as brain imaging through magnetic resonance imaging were also performed. Dopamine transporter SPECT scans were available for most patients with suspected DLB [19].

\section{Classification of Frailty}

Collected information was employed to estimate frailty through the FI, which was blinded for clinical diagnosis. Our FI included 35 deficits:

(1) Inability to cook for themselves, (2) Inability to keep personal hygiene (shaving, hair combing, and dental hygiene), (3) Inability to go to the bathroom by themselves, (4) Inability to use the phone, (5) Inability to go shopping, (6) Inability to manage their finances, (7) Inability to take own medicines, (8) Inability to manage financial responsibilities (pay taxes), (9) Upper gastrointestinal complaints, (10) Lower gastrointestinal complaints, (11) Urinary system deficits, (12) Osteomuscular complaints, (13) Renal problems, (14) Low serum albumin, (15) Hypoglycemia, (16) Cardiac diseases (Arrhythmias, Myocardial infarction, Congestive heart failure), (17) Hypertension, (18) Diabetes Mellitus, (19) COPD, (20) Hypothyroidism, (21) Epilepsy, (22) Stroke or Transient Ischemic Attack, (23) Gait disturbances, (24) Tremor, (25) Smoking, (26) Glaucoma or other ophthalmologic diseases, (27) Delirium, (28) Hearing problems, (29) Aphasia, (30) Arthropathies, (31) Agitation or aggression, (32) Depression, (33) Anxiety, (34) Apathy, and (35) Falls.

Each item was rated as 0 (if no deficit was present) or 1 (if a real deficit was present), deficits from 1 to 13 had intermediate scores such as $0,0.33,0.66$, and 1 according to severity. The score for each item was added and then divided by 35 in order to obtain a global score for the index. The cutoff value used to define frailty was established at $>0.25$ [4].

\section{Statistical Analysis}

Descriptive analyses are presented through frequencies and percentages for categorical variables, while SDs and means are used for continuous variables. For bivariate comparative analyses, $\chi^{2}$-tests or Student $t$ tests were used as appropriate, depending on the distribution 
Table 1. Descriptive and bivariate analysis

\begin{tabular}{|c|c|c|c|c|}
\hline & Non-frail & Frail & $p$ value & Total \\
\hline & $n(\%)$ or mean \pm SD & $n(\%)$ or mean \pm SD & & $n(100 \%)$ or mean \pm SD \\
\hline Age, years & $75.01 \pm 7.95$ & $79.21 \pm 5.40$ & 0.0009 & $76.10 \pm 7.59$ \\
\hline$\leq 69$ & 96.55 (28) & 3.45 (1) & 0.003 & 29 \\
\hline $70-79$ & $75.86(66)$ & $24.14(21)$ & & 87 \\
\hline$\geq 80$ & $64.18(43)$ & $35.82(24)$ & & 67 \\
\hline Gender & & & 0.5196 & \\
\hline Male & $47(68.12)$ & $22(31.88)$ & & 69 \\
\hline Female & $91(77.78)$ & $26(22.22)$ & & 117 \\
\hline MMSE & $23.81 \pm 2.61$ & $22.93 \pm 2.62$ & 0.0458 & $23.59 \pm 2.63$ \\
\hline $\mathrm{AD}$ & $94(81.03)$ & $22(18.97)$ & 0.006 & 116 \\
\hline DLB & $44(62.86)$ & $26(37.14)$ & & 70 \\
\hline Total & $138(74.19)$ & $48(25.81 \%)$ & & 186 \\
\hline FI & $0.15 \pm 0.079$ & $0.21 \pm 0.01$ & $<0.001$ & $0.18 \pm 0.094$ \\
\hline
\end{tabular}

MMSE, Mini-Mental Status Examination; FI, frailty index; AD, Alzheimer's disease; DLB, dementia with lewy bodies.

of each variable. Multivariate logistic regression models were fitted in order to obtain an OR with $95 \% \mathrm{CI}$ for the association between frailty and the independent variable (AD or DLB). The results were then adjusted for sex, age, and MMSE. The level of statistical significance was set at $p<0.05$. All data were analyzed using STATA 15 .

This study was approved by the regional Ethics Committee (2010/633) and the Norwegian authorities for the collection of medical data. All data were handled in accordance with national health and data privacy protocols. All participants signed an informed consent form before inclusion in the study.

\section{Results}

The cohort included 186 patients with mean age $76.10 \pm 7.59,116$ with $\mathrm{AD}$ and 70 with DLB. Demographic and clinical characteristics for frail DLB and AD patients are shown in Table 1. Frailty was associated with higher age ( $79.21 \pm 5.40$ vs. $75.01 \pm 7.95, p$ value $\leq 0.001)$, male gender $(31.88 \%$ vs. women $22.22 \%, p$ value $=0.51$ and lower MMSE scores $(22.93 \pm 2.62$ vs. $23.81 \pm 2.61, p$ value $=0.045$; Table 1 ) .

The global frequency of frailty was $25.81 \%(n=48)$. In DLB, $37.14 \%(n=26)$ of the subjects were frail, compared to $18.97 \%(n=22)$ of those with $\mathrm{AD}(p=0.006$; Table 1 and Fig. 1). The adjusted multivariate analysis revealed an OR of 2.45 (1.15-5.23) for frailty in subjects with DLB when taking AD as the reference group. This association was statistically significant ( $p$ value 0.020 ; Table 2 ).

\section{Discussion}

We found a high general prevalence of frailty within the sample, with an even greater prevalence in those living with DLB. The odds for being frail were more than 2 times higher in DLB patients when compared with subjects with AD. 
Table 2. Multivariate analysis

\begin{tabular}{|c|c|c|c|c|}
\hline & \multicolumn{2}{|l|}{ Unadjusted } & \multicolumn{2}{|l|}{ Adjusted } \\
\hline & OR $(95 \% \mathrm{CI})$ & $p$ value & OR $(95 \% \mathrm{CI})$ & $p$ value \\
\hline $\mathrm{AD}$ & Ref. & & Ref. & \\
\hline DLB & $2.52(1.29-4.94)$ & 0.007 & $2.45(1.15-5.23)$ & 0.020 \\
\hline Age, years & - & & $1.11(1.04-1.18)$ & 0.001 \\
\hline Gender & - & & $0.61(0.28-1.31)$ & 0.211 \\
\hline MMSE & - & & $0.94(0.82-1.07)$ & 0.381 \\
\hline
\end{tabular}

Model adjusted by sex, age and score in the MMSE.

AD, Alzheimer's disease; DLB, dementia with lewy bodies; MMSE, Mini-Mental Status Examination.

Fig. 1. Distribution of people with $\mathrm{AD}$ and DLB according to the FI. DLB, dementia with Lewy bodies; AD, Alzheimer's disease; FI, frailty index.

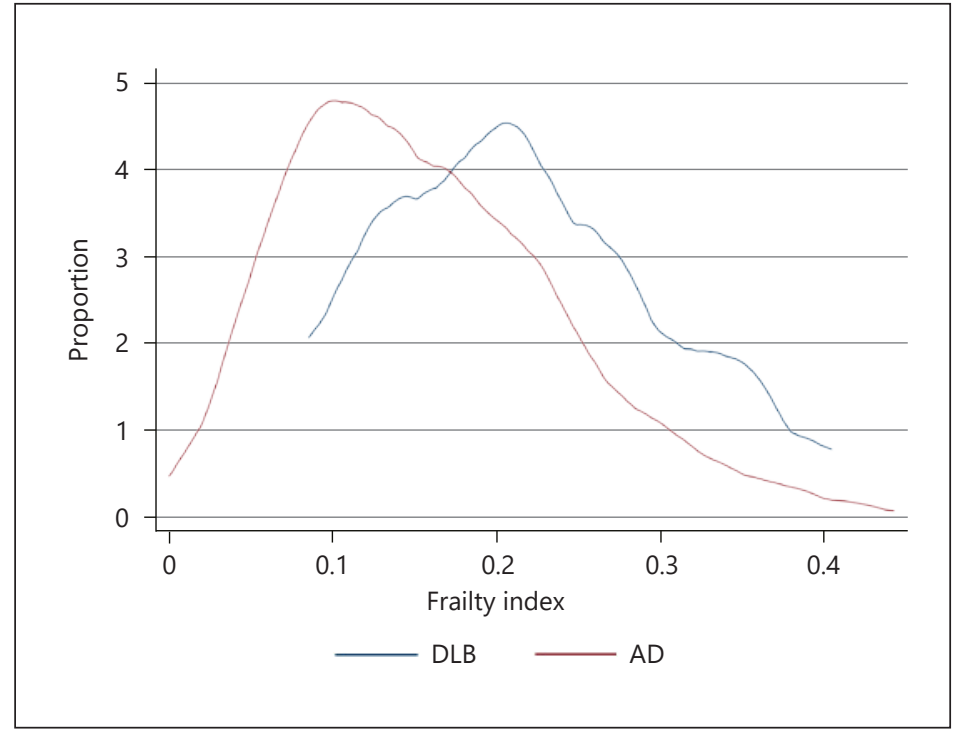

The trajectories of frailty and dementia share multiple etiologies, features, and related factors. However, in contrast with AD, DLB patients are more prone to be frail due to the symptoms of the disease per se. (i.e., executive function impairment, decreased mobility, motor and non-motor symptoms, psychiatric and autonomous symptoms) and due to the consequences of these manifestations (poor adherence and adverse effects to medications, polypharmacy, worsening of comorbidities, need for care, falls, geriatric syndromes, among others) [24]. Our research group has previously reported high mortality in DLB, which we can now infer, may be associated with the high prevalence of frailty [25].

For the purpose of this study, we used the FI because in this context it reflects the biological nature of frailty more than other approaches based mainly on physical variables and measurements, which can be biased by the illness itself. Furthermore, dementias in general have been long seen as diseases that are limited to the brain, focusing on cognitive and mental symptoms. However, and more importantly in DLB, other systemic manifestations and their consequences are very relevant and are usually under-detected or considered to be normal age-related changes [24]. All of these factors could lead to a greater and faster fragilization process. 
It is also relevant to mention that people living with frailty are more likely to develop dementia due to several factors including inflammation and endothelial damage [26-28]. Thus, targeting frailty as a potentially modifiable factor in the early trajectory of dementia seems imperative. The prevalence of frailty found in our study is similar to previous reports on AD [29]. A recent systematic review reported the prevalence of frailty within people with AD ranging from 11.1 to $50 \%$, with a pooled prevalence of $31.9 \%$ in mild and moderate stages [14]. However, the association between cognitive impairment and frailty had only been reported in AD.

It is important to highlight that people included in this analysis had been recently diagnosed with mild dementia, which leads us to conclude that in many cases the frailty process starts much before the onset of dementia, and efforts should be made toward prodromal or early detection of both neurodegenerative diseases and frailty.

Our study is at risk for potential recruitment bias because of referrals by primary care physicians, which may have led to an increased number of patients with complicated dementia or neuropsychiatric symptoms. However, referral centers were invited to refer any patients with suspected dementia, and patients were included from psychiatric, neurologic, and geriatric clinics. MMSE is language and memory dominant and thus less sensitive to the earliest changes in DLB patients, although the sensitivity of MMSE is comparable to other screening instruments when approaching moderate dementia levels [30]. In addition, we also used the CDR to compare dementia severity, which is independent of the cognitive impairment profile. Also, healthy controls were not included for comparison. Strengths of the study include careful and rigorous diagnostic procedures, assessment through structured instruments, longitudinal design, and high postmortem accuracy in the 56 cases with neuropathological diagnosis $[19,23]$. To the best of our knowledge, this is the first study of its kind to address the association between frailty and DLB.

\section{Conclusion}

The prevalence of frailty at the time of diagnosis of dementia is high. Clinicians, particularly primary care practitioners, are thus encouraged to identify dementia earlier in its trajectory for timely and prompt interventions. Understanding frailty in DLB may guide management and improve prognosis. Nonetheless, better tools are required in order to allow early or prodromal detection of disease and prevent or stop the fragilization process, particularly in diseases such as DLB where frailty has shown to be prevalent and has been unexplored.

\section{Statement of Ethics}

This study was approved by the regional Ethics Committee (2010/633) and the Norwegian authorities for the collection of medical data, and received financial support from the regional health authorities of Western Norway, Helse Vest. All data were handled and kept in accordance with national health and data privacy protocols. All participants signed an informed consent form before inclusion in the study.

\section{Disclosure Statement}

The authors have no potential conflicts of interest to declare regarding research, authorship, and/or publication of this article. 


\section{Funding Sources}

This paper represents independent research supported by the Norwegian government, through hospital owner Helse Vest (Western Norway Regional Health Authority). Also, funded by the National Institute for Health Research (NIHR) Biomedical Research Centre at South London and Maudsley NHS Foundation Trust and King's College London. The views expressed are those of the author(s) and not necessarily those of the NHS, the NIHR or the Department of Health and Social Care.

\section{Author Contributions}

M.G.B., H.S.: Conception and design, analysis and interpretation of data, manuscript preparation, final approval of the version to be published. C.E.: Conception and design, manuscript preparation, final approval of the version to be published. M.U.P-.Z.: Conception and design, analysis and interpretation of data, final approval of the version to be published. D.A.: Conception and design, analysis and interpretation of data, manuscript preparation, final approval of the version to be published, DEM-VEST study PI.

\section{References}

1 Avila-Funes JA, Medina-Campos RH, Tamez-Rivera O, Navarrete-Reyes AP, Amieva H, Aguilar-Navarro S. Frailty is associated with disability and recent hospitalization in community-dwelling elderly: the coyoacan cohort. J Frailty Aging. 2014;3(4):206-10.

2 Fried LP, Ettinger WH, Lind B, Newman AB, Gardin J; Cardiovascular Health Study Research Group. Physical disability in older adults: a physiological approach. J Clin Epidemiol. 1994 Jul;47(7):747-60.

3 Rockwood K, Mitnitski A. Frailty defined by deficit accumulation and geriatric medicine defined by frailty. Clin Geriatr Med. 2011 Feb;27(1):17-26.

4 Searle SD, Mitnitski A, Gahbauer EA, Gill TM, Rockwood K. A standard procedure for creating a frailty index. BMC Geriatr. 2008 Sep;8(1):24.

5 Samper-Ternet R, Reyes-Ortiz C, Ottenbacherc K, Cano C. Frailty and Sarcopenia in Colombia: Results from the SABE Bogotá Study. European geriatric medicine. 2015 Sep;6(1):S84.

6 Siriwardhana DD, Hardoon S, Rait G, Weerasinghe MC, Walters KR. Prevalence of frailty and prefrailty among community-dwelling older adults in low-income and middle-income countries: A systematic review and metaanalysis. BMJ Open. 2018;8:e018195.

7 Livingston G, Sommerlad A, Orgeta V, Costafreda SG, Huntley J, Ames D, et al. Dementia prevention, intervention, and care. Lancet. 2017 Dec;390(10113):2673-734.

8 Prince MJ, Wu F, Guo Y, Gutierrez Robledo LM, O'Donnell M, Sullivan R, et al. The burden of disease in older people and implications for health policy and practice. Lancet. 2015 Feb;385(9967):549-62.

9 WHO. Dementia a public health priority. United Kingdom: World Health Organization; 2012.

10 Shimada H, Makizako H, Lee S, Doi T, Lee S, Tsutsumimoto K, et al. Impact of cognitive frailty on daily activities in older persons. J Nutr Health Aging. 2016;20(7):729-35.

11 Solfrizzi V, Scafato E, Frisardi V, Sancarlo D, Seripa D, Logroscino G, et al.; Italian Longitudinal Study on Aging Working Group. Frailty syndrome and all-cause mortality in demented patients: the Italian Longitudinal Study on Aging. Age (Dordr). 2012 Apr;34(2):507-17.

12 Bunn F, Goodman C, Burn AM. Multimorbidity and frailty in people with dementia. Nurs Stand. 2015 Sep; $30(1): 45-50$.

13 Neale R, Brayne C, Johnson AL; Medical Research Council Cognitive Function and Ageing Study Writting Committee. Cognition and survival: an exploration in a large multicentre study of the population aged 65 years and over. Int J Epidemiol. 2001 Dec;30(6):1383-8.

14 Kojima G, Liljas A, Iliffe S, Walters K. Prevalence of frailty in mild to moderate alzheimer's disease: A systematic review and meta-analysis. Curr Alzheimer Res. 2017;14(12):1256-63.

15 McKeith IG, Boeve BF, Dickson DW, Halliday G, Taylor JP, Weintraub D, et al. Diagnosis and management of dementia with Lewy bodies: fourth consensus report of the DLB Consortium. Neurology. 2017 Jul;89(1): 88-100. 
16 Zweig YR, Galvin JE. Lewy body dementia: the impact on patients and caregivers. Alzheimers Res Ther. 2014 Apr;6(2):21-21.

17 Mueller C, Ballard C, Corbett A, Aarsland D. The prognosis of dementia with Lewy bodies. Lancet Neurol. 2017 May; 16(5):390-8.

18 Müller F, Naharro M, Carlsson GE. What are the prevalence and incidence of tooth loss in the adult and elderly population in Europe? Clin Oral Implants Res. 2007 Jun;18 Suppl 3:2-14.

19 Aarsland D, Rongve A, Nore SP, Skogseth R, Skulstad S, Ehrt U, et al. Frequency and case identification of dementia with Lewy bodies using the revised consensus criteria. Dement Geriatr Cogn Disord. 2008;26(5): 445-52.

20 McKhann G, Drachman D, Folstein M, Katzman R, Price D, Stadlan EM. Clinical diagnosis of alzheimer's disease: report of the nincds-adrda work group under the auspices of department of health and human services task force on alzheimer's disease. Neurology. 1984 Jul;34(7):939-44.

21 McKeith IG, Dickson DW, Lowe J, Emre M, O’Brien JT, Feldman H, et al.; Consortium on DLB. Diagnosis and management of dementia with Lewy bodies: third report of the DLB Consortium. Neurology. 2005 Dec;65(12): 1863-72.

22 Folstein MF, Folstein SE, McHugh PR. "Mini-mental state”. A practical method for grading the cognitive state of patients for the clinician. J Psychiatr Res. 1975 Nov;12(3):189-98.

23 Skogseth R, Hortobágyi T, Soennesyn H, Chwiszczuk L, Ffytche D, Rongve A, et al. Accuracy of clinical diagnosis of dementia with lewy bodies versus neuropathology. Journal of Alzheimer's disease. J Alzheimers Dis. 2017; 59(4):1139-52.

24 Borda MG, Zuluaga M, Garcia-Cifuentes E, et al. Lewy body dementia in frailty, a call for a geriatrician. Moj gerontol ger. 2017;2(2):222-223. 2017

25 Vik-Mo AO, Giil LM, Ballard C, Aarsland D. Course of neuropsychiatric symptoms in dementia: 5-year longitudinal study. Int J Geriatr Psychiatry. 2018 Oct;33(10):1361-9.

26 Amin J, Williams T, Teeling J, Dorey R, Williams D, Tommasino E, et al. Inflammation in dementia with lewy bodies. Alzheimers Dement. 2017;13(7):P594-P595.

27 Enciu AM, Popescu BO. Is there a causal link between inflammation and dementia? BioMed Res Int. 2013;2013: 316495.

28 Kulmala J, Nykänen I, Mänty M, Hartikainen S. Association between frailty and dementia: a population-based study. Gerontology. 2014;60(1):16-21.

29 Sampson EL. Frailty and dementia: common but complex comorbidities. Aging Ment Health. 2012;16(3):26972.

30 Mitchell AJ. A meta-analysis of the accuracy of the mini-mental state examination in the detection of dementia and mild cognitive impairment. J Psychiatr Res. 2009 Jan;43(4):411-31. 